\title{
Increased risk of venous thromboembolism in patients with bullous pemphigoid
}

\section{The INVENTEP (INcidence of VENous ThromboEmbolism in bullous Pemphigoid) study}

\begin{abstract}
Massimo Cugno 15; Angelo V. Marzano2§; Paolo Bucciarelli3; Ylenia Balice ${ }^{2}$; Giuseppe Cianchini4; Pietro Quaglino5; Piergiacomo Calzavara Pinton ${ }^{6}$; Marzia Capronii; Mauro Alaibac ${ }^{8}$; Clara De Simone ${ }^{9}$; Annalisa Patrizi ${ }^{10}$; Emanuele Cozzani ${ }^{11}$; Manuela Papini12; Alberto Tedeschi'; Emilio Berti²; Frits R. Rosendaal13; for the INVENTEP Study Group*

${ }^{1}$ Internal Medicine, Department of Pathophysiology and Transplantation, University of Milan, Fondazione IRCCS Ca' Granda Ospedale Maggiore Policlinico, Milan, Italy; ${ }^{2}$ Dermatology Unit, Department of Pathophysiology and Transplantation, University of Milan, Fondazione IRCCS Ca' Granda Ospedale Maggiore Policlinico, Milan, Italy; ${ }^{3}$ A. Bianchi Bonomi Hemophilia and Thrombosis Center, Fondazione IRCCS Ca' Granda Ospedale Maggiore Policlinico, Milan, Italy; ${ }^{4}$ Department of Immunodermatology, Istituto Dermopatico dell'Immacolata, Istituto di Ricovero e Cura a Carattere Scientifico (IRCCS), Rome, Italy; ${ }^{5}$ Department of Medical Sciences Dermatologic Division, University of Turin, Turin, Italy; ${ }^{6}$ Department of Dermatology, University Hospital Spedali Civili, Brescia, Italy; ${ }^{7}$ Department of Surgery and Translational Medicine, Section of Dermatology, University of Florence, Florence, Italy; ${ }^{8}$ Unit of Dermatology, University of Padua, Padua, Italy; ${ }^{9}$ Department of Dermatology, Catholic University of the Sacred Heart, Rome, Italy; ${ }^{10}$ Dermatology, Department of Specialized, Experimental and Diagnostic Medicine, Sant'Orsola -Malpighi Hospital, University of Bologna, Italy; ${ }^{11}$ IRCCS AOU San Martino Di.S.Sal., Section of Dermatology, University of Genoa, Genoa, Italy; ${ }^{12}$ Department of Surgical and Biomedical Sciences, Dermatology Unit of Terni, University of Perugia, Terni, Italy; ${ }^{13}$ Department of Clinical Epidemiology, and Department of Thrombosis and Hemostasis, Leiden University Medical Center, Leiden, The Netherlands
\end{abstract}

\begin{abstract}
Summary
Activation of blood coagulation has been demonstrated in bullous pemphigoid (BP), a rare autoimmune blistering disease, potentially leading to a prothrombotic state. In order to evaluate the incidence of venous thromboembolism (VTE) in BP, a cohort study was carried out on 432 BP patients ( $59 \%$ females; median age 76 years, interquartile range [IQR]: 68-82). At diagnosis, autoimmune bullous skin disorder intensity score (ABSIS) was calculated. VTE incidence was standardised with rates of the general population. Multivariable Cox proportional hazard model was used to estimate the hazard ratio of VTE according to ABSIS and concomitant risk factors. During a median follow-up of 4.2 years, 31 objectively-diagnosed VTE events were recorded. The incidence rate of VTE (per 1000 patient-years) was 17.2 overall (95\% confidence interval [Cl]: 11.1-23.2), $56.7(95 \% \mathrm{Cl}$ :
\end{abstract}

33.0-80.4) during acute phase (22 VTE) and 6.3 (95\%Cl: 2.8-11.3) during remission (9 VTE). The standardised incidence ratio was 4.06 (95\%Cl: 2.73-5.65), higher during the acute phase $(14.86,95 \% \mathrm{Cl}$ : $9.20-21.88)$ than during remission $(1.48,0.66-2.63)$. The adjusted hazard ratio of VTE was $2.74(95 \% \mathrm{Cl}: 1.07-7.04)$ for ABSIS $>48$ vs ABSIS $<28$, and $2.56(95 \% \mathrm{Cl}: 1.00-6.70)$ in patients with $\geq 2$ concomitant risk factors. In conclusion, BP patients have a 15 -fold increased VTE risk during acute phase, proportional to disease severity and heightened by concomitant risk factors.

\section{Keywords}

Bullous pemphigoid, autoimmune bullous skin disorders, thromboembolism, thrombotic risk

Financial support:

This work was supported by "Ricerca corrente", Fondazione IRCCS Ca' Granda, Ospedale Maggiore Policlinico, Milano, Italy. The funder had no role in study design, data collection and analysis, decision to publish, or preparation of the manuscript.

Received: April 15, 2015

Accepted after major revision: June 19, 2015

Epub ahead of print: August 6, 2015

http://dx.doi.org/10.1160/TH15-04-0309

Thromb Haemost 2016; 115: 193-199

\section{Introduction}

Bullous pemphigoid (BP) is a rare autoimmune blistering disease that typically occurs in the elderly (1) and carries a high risk of death (2), mainly due to sepsis and cardiovascular events (3). A recent study carried out in Switzerland reported an incidence of 12.1 new BP cases per million people per year which increased up to 50 cases per million in the eighth decade of life (4). It involves the skin and rarely the mucous membranes and is characterised by the presence of blisters usually surrounded by erythematous-edematous lesions ( Figure 1). Autoantibodies against two hemidesmosomal antigens (BP 180 and BP 230) play an important role in the pathogenesis of $\mathrm{BP}$ as well as complement activation and leukocyte infiltration (1). Inflammatory cells, particularly autoreactive T 


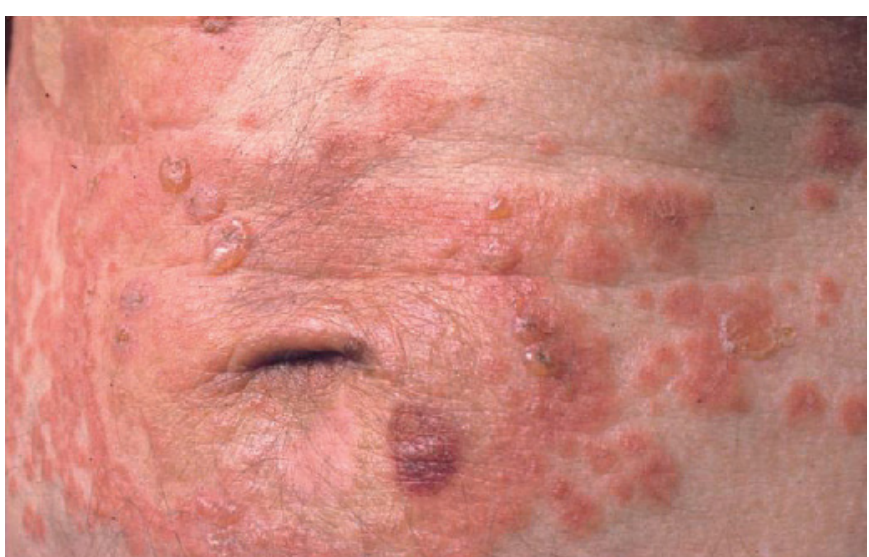

Figure 1: Typical cutaneous manifestations in a patient with bullous pemphigoid: tense blisters surrounded by erythematousoedematous lesions.

cells and eosinophils, participate in blister formation by producing and releasing a number of cytokines and soluble factors that amplify and maintain tissue damage (5). The inflammatory response induces activation of blood coagulation which is involved both locally, by amplifying the inflammatory network in lesional skin, and systemically, by leading to a prothrombotic state (6-11). In a previous study on the involvement of blood coagulation in the pathophysiology of BP, an apparent high frequency of various thrombotic complications was incidentally found in a series of $130 \mathrm{BP}$ patients (6). In particular, venous thromboembolism (VTE) seemed to occur more frequently (6) than in the elderly general population (12). Although cardiovascular complications are frequently observed in BP patients and are regarded as important causes of death $(3,13,14)$, to date there are no studies on the incidence of VTE in BP, except for the study by Langan et al. (15), who investigated the main acute medical conditions associated with $\mathrm{BP}$, finding an increased risk only for pulmonary embolism and pneumonia.

In the present multicentre cohort study, the primary objectives were to estimate the incidence of VTE in patients with a first diagnosis of BP (overall and separately in the acute phase of disease and at remission), and to evaluate whether the risk of VTE is associated with the inflammatory burden typical of BP. A secondary objective was to assess the impact of concomitant risk factors on the risk of VTE.

\section{Patients and methods Study population}

The study cohort included 432 patients consecutively referred for the diagnosis of BP at 11 Italian Centres (Milan (2 centres), Rome ( 2 centres), Florence, Genoa, Turin, Bologna, Padua, Terni and Brescia) between January 1, 2006 and January 1, 2011. The diagnosis of BP was based on clinical, histopathological and immunopathological criteria, and was established on the basis of typical clinical findings (blisters/erosions of the skin and less frequently of the mucous membranes, and/or erythematous-oedematous skin lesions), as well as histopathological and immunopathological criteria $(6,7)$. Histology showed dermoepidermal detachments and a dermal mononuclear cell inflammatory infiltrate rich in eosinophils. Direct immunofluorescence examination of the perilesional skin revealed the linear deposition of $\operatorname{IgG}$ or $\mathrm{C} 3$ in the basement membrane zone (BMZ) in all patients. Circulating anti-BMZ autoantibodies were detected by means of IIF on monkey oesophagus substrates performed on $1 \mathrm{~mol} \mathrm{~L}^{-1} \mathrm{NaCl}$ human split skin, which disclosed autoantibody binding on the epidermal side in all patients. Finally, circulating anti-BP180 or anti-BP 230 autoantibodies were found by ELISA.

At diagnosis, the inflammatory burden typical of BP, expression of its severity, was evaluated using the autoimmune bullous skin disorder intensity score (ABSIS), the most commonly used and validated score for patients with blistering skin diseases (16). Briefly, ABSIS focused on the extent of the affected area and on the quality of the skin lesions, the extent of which was assessed by the "rule of nine". Areas equal to 9\% (or its multiple) of the total body surface area were assessed; the palm of the patient's hand (and areas of equivalent size) was set at $1 \%$ of body surface area. The extent of the affected area (in \%) was then multiplied by a weighting factor ( 1.5 for blisters and erosive/exudative lesions, 1 for erosive/ dry lesions, and 0.5 for re-epithelised lesions).

For all patients demographic and clinical data (including ongoing therapies and the presence of concomitant risk factors for VTE) were collected at the baseline visit. We considered as concomitant risk factors for VTE the following: recent ( $<3$ months) surgery, trauma or leg fractures, bed rest for more than five days, previous episodes of VTE, obesity (BMI $>30 \mathrm{~kg} / \mathrm{m}^{2}$ ) and cancer. Obesity and cancer were considered as permanent risk factors, and the others as transient risk factors. VTE included lower-limb deepvein thrombosis (no compression of the calf trifurcation or more proximal on ultrasonography or an intraluminal filling defect on venography) or pulmonary embolism (perfusion lung scan or spiral computerised tomography scan). After BP diagnosis, each patient was treated with systemic corticosteroids (oral prednisone at initial daily dose of $\leq 0.7 \mathrm{mg} / \mathrm{kg}$ ). During the follow-up, one visit every month was scheduled for the first three months and then on clinical grounds. At each follow-up visit, the disease status of the patient was recorded (acute phase or remission) and therapy modified accordingly. In case of worsening of disease status (i.e. occurrence of new blisters or extension of previous skin lesions), the scheduled visit was made in advance. Moreover, exposure to concomitant risk factors in the period between two visits was checked and, in case of hospital admissions for any reason (including a VTE episode) or death, medical charts were reviewed. Patients were invited to report any symptom of VTE, in order to perform the appropriate diagnostic work-up, and only objectivelydiagnosed VTE episodes were considered in this study. Superficial thrombophlebitis, which are often clinically diagnosed, were only described but not included in the analysis. The study was approved by the Institutional Review Board of the coordinating hospital and all patients gave their informed consent to participate into the study. 
Figure 2: Schematic representation of how person-time was partitioned into acute phase and remission for each patient with bullous pemphigoid. Each row represents a possible scenario of a patient during follow-up at each scheduled visit. At diagnosis, all patients with bullous pemphigoid are in the acute phase, then they could alternate some periods of remission and some periods of disease relapse. In case a remission is achieved after a period of acute phase, the person-time between the two visits is split in two equal portions for acute phase and remission. On the contrary, in case of relapse after a period of remission, all the person-time between the two visits is assigned to the remission phase, because we suppose that the time elapsed between the relapse (start of a new acute phase) and the subsequent visit is very short. PT-YRS=patient-years.

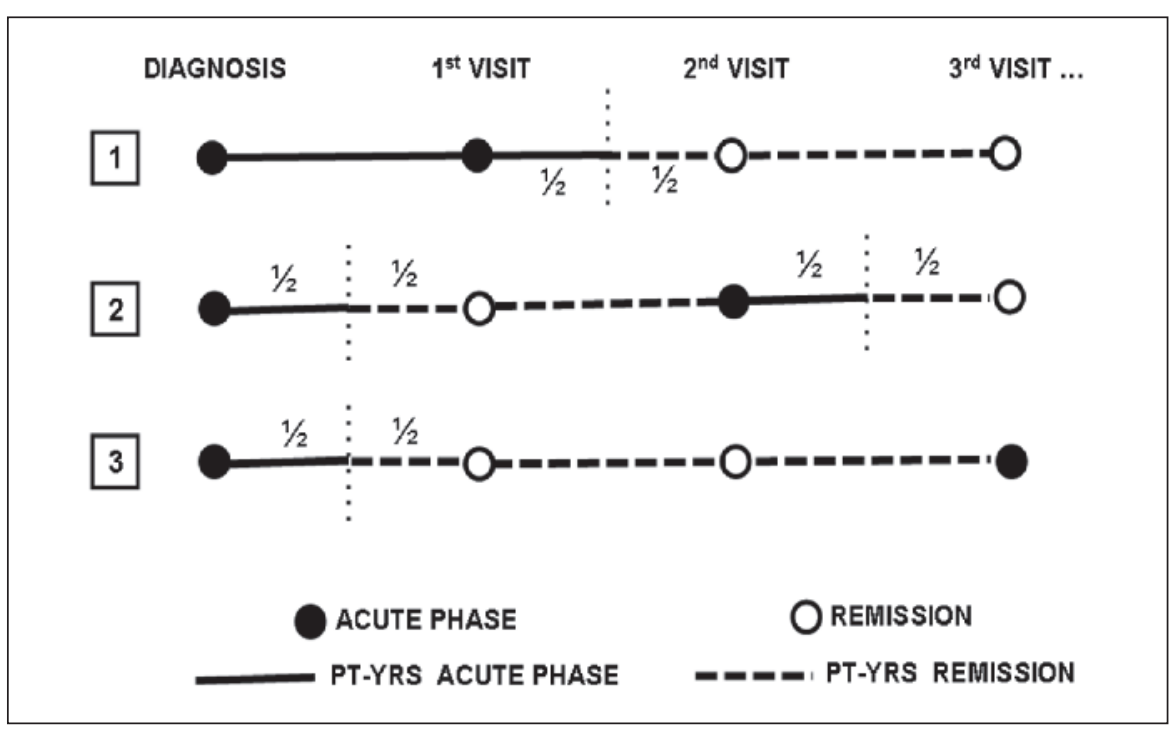

\section{Statistical analysis}

Continuous variables were expressed as medians and interquartile range (IQR), and categorical variables as counts and percentages. The follow-up period lasted from the date of BP diagnosis until the date of a VTE event, death, last visit or July $1^{\text {st }} 2013$ (administrative censoring), whichever came first. The overall incidence rate of VTE was calculated as the number of VTE episodes divided by the total number of patient-years of exposure, and the $95 \%$ confidence interval (CI) was calculated under the Poisson distribution assumption. Separate incidence rates of VTE during the acute phase of $\mathrm{BP}$ and at remission were calculated by partitioning the person-time of each patient into fractions corresponding to each disease phase ( $>$ Figure 2). All incidence estimates were standardised to those of the general population according to age and sex (17) through the indirect method. The person-years each individual of the study population contributed to the follow-up were split in the different age categories separately for men and women. The obtained standardised incidence ratio, and its $95 \% \mathrm{CI}$, is the ratio between observed and expected VTE, and represents a measure of the magnitude of the VTE incidence in the study population relative to that in the general population of same age and sex (i.e. a standardised incidence ratio higher than 1 means that the incidence of VTE in individuals with BP is higher than that expected in a population with the age-year specific rates of the general population and the person-years distribution observed in the study population). The cumulative incidence of VTE was calculated with the Kaplan-Meier method. The hazard ratio (HR) of VTE according to ABSIS, adjusted for age (continuous), sex ( $0=$ female, $1=$ male) and concomitant risk factors $(0,1, \geq 2)$, was estimated together with its $95 \% \mathrm{CI}$ in a multivariable Cox proportional hazard model. ABSIS was first kept continuous and the possible presence of non-linear effects of the ABSIS distribution on the log of the relative hazard of VTE was evaluated using a restricted cubic spline function with three knots, and tested by using the Wald test. Then, ABSIS was divided into tertiles of its distribu- tion $(<28,28-48,>48)$ and the HR of VTE for each tertile was calculated taking the lowest one as reference. In a second multivariable Cox proportional hazard model we looked also at a possible interaction between ABSIS (first continuous and then divided in two categories ( $>48$ vs $\leq 48)$ ) and concomitant risk factors ( $\geq 2$ vs 0 or 1): the HR of VTE, adjusted for age and sex, for each of the four possible combinations was estimated taking the category of ABSIS $<28$ and no or one risk factor as reference. A sensitivity analysis was carried out in which missing values for ABSIS were substituted with values obtained from linear interpolation by multiple imputation. For all statistical analyses, the SPSS PC statistical package, release 20.0 (IBM SPSS Inc., Chicago, IL, USA) and the

Table 1: Baseline characteristics of the study cohort.

\begin{tabular}{|l|l|}
\hline Patient characteristics & Values \\
\hline Total number & 432 \\
\hline Sex (M / F) & 176 / 256 \\
\hline Age at diagnosis (years), median (IQR) & $76(68-82)$ \\
\hline Follow-up (years), median (IQR) & $4.20(2.93-6.00)$ \\
\hline Deaths, $n$ (\%) & $92(21)$ \\
\hline Lost at follow-up, $n(\%)$ & $10(2.3)$ \\
\hline ABSIS, median (IQR) ${ }^{*}$ & $39(22-63)$ \\
\hline Concomitant risk factors, $n(\%)^{\dagger}$ & - \\
\hline none & $279(65)$ \\
\hline surgery & $63(15)$ \\
\hline trauma / bed rest & $74(17)$ \\
\hline obesity & $27(6)$ \\
\hline cancer & $52(12)$ \\
\hline $\begin{array}{l}\text { IQR }^{+} \text {interquartile range; ABSIS= autoimmune bullous skin disorder intensity } \\
\text { score. }{ }^{*} \text { Data not available for } 16 \text { patients. }{ }^{\dagger} \text { Some patients were exposed to } \\
\text { more than one risk factor. }\end{array}$ \\
\hline
\end{tabular}


Table 2: Standardisation of VTE incidence rates of the study population of patients with bullous pemphigoid (BP) according to sex and age, with the general population as reference.

\begin{tabular}{|c|c|c|c|c|c|}
\hline \multicolumn{6}{|c|}{ BP patients } \\
\hline Sex & $\begin{array}{l}\text { Age } \\
\text { (years) }\end{array}$ & $\begin{array}{l}\text { Incidence } \\
\text { rate gen- } \\
\text { eral popu- } \\
\text { lation * }\end{array}$ & $\begin{array}{l}\text { Study } \\
\text { population } \\
\text { (patient- } \\
\text { years) }\end{array}$ & $\begin{array}{l}\text { Ob- } \\
\text { served } \\
\text { VTE }\end{array}$ & $\begin{array}{l}\text { Expected } \\
\text { VTE }\end{array}$ \\
\hline \multirow[t]{6}{*}{ Men } & $35-44$ & 0.36 & 12 & 0 & 0 \\
\hline & $45-54$ & 0.76 & 32 & 0 & 0.02 \\
\hline & $55-64$ & 1.53 & 62 & 1 & 0.10 \\
\hline & $65-74$ & 2.94 & 229 & 1 & 0.67 \\
\hline & $75-84$ & 5.46 & 266 & 5 & 1.45 \\
\hline & $85+$ & 5.55 & 143 & 3 & 0.79 \\
\hline \multirow[t]{6}{*}{ Women } & $35-44$ & 0.44 & 15 & 0 & 0 \\
\hline & $45-54$ & 1.00 & 52 & 0 & 0.05 \\
\hline & $55-64$ & 1.30 & 120 & 0 & 0.16 \\
\hline & $65-74$ & 2.14 & 226 & 6 & 0.48 \\
\hline & $75-84$ & 5.17 & 408 & 10 & 2.11 \\
\hline & $85+$ & 7.40 & 243 & 5 & 1.80 \\
\hline Overall & & & & 31 & 7.63 \\
\hline
\end{tabular}

* per 1,000 patient-years. Data obtained from Reference 17. statistical software R, release 3.0.0 (R Foundation for Statistical Computing, Vienna, Austria) were used.

\section{Results}

Of the 432 BP patients, 256 (59\%) were women, and the median age was 76 years (IQR 68-82). Table 1 shows the baseline characteristics of the study cohort. The median follow-up was 4.2 years (IQR 2.9-6.0), for a total of 1,807 patient-years (388 for the acute phase and 1,419 for remission). No patient reported a previous episode of VTE, making an extensive screening for genetic prothrombotic conditions not mandatory. No patient was taking anticoagulants or was under hormone therapy at the time of BP diagnosis. The causes of death were the following: heart failure $(\mathrm{n}=15)$, stroke ( $\mathrm{n}=12)$, cancer $(\mathrm{n}=9)$, sepsis $(\mathrm{n}=8)$, acute myocardial infarction $(n=8)$, pulmonary embolism $(n=4)$, undefined $(n=36)$. The 4 deaths related to pulmonary embolism occurred suddenly $(n=3)$ or within one year $(\mathrm{n}=1)$ since its diagnosis, and two deaths ( 1 for stroke and 1 for cancer) occurred one to two years after the episode of VTE. No substantial difference in the ABSIS was evident between different Centres, and ABSIS positively correlated with anti-BP180 autoantibodies (Spearman's rho 0.55; $\mathrm{p}<0.0001$ ).

Thirty-one objectively-diagnosed VTE episodes (20 lower-limb deep vein thromboses, 9 deep vein thromboses with pulmonary embolism and 2 isolated pulmonary embolism) were recorded. All deep-vein thromboses involved the proximal veins. Three additional cases of superficial thrombophlebitis were described. The overall incidence rate of VTE (per 1,000 patient-years) was 17.2 (95\%CI: 11.1-23.2). This figure was 56.7 (95\%CI: 33.0-80.4) during the acute phase (22 VTE) and 6.3 (95\%CI: 2.8-11.3) during remission (9 VTE). After standardisation with rates of the general

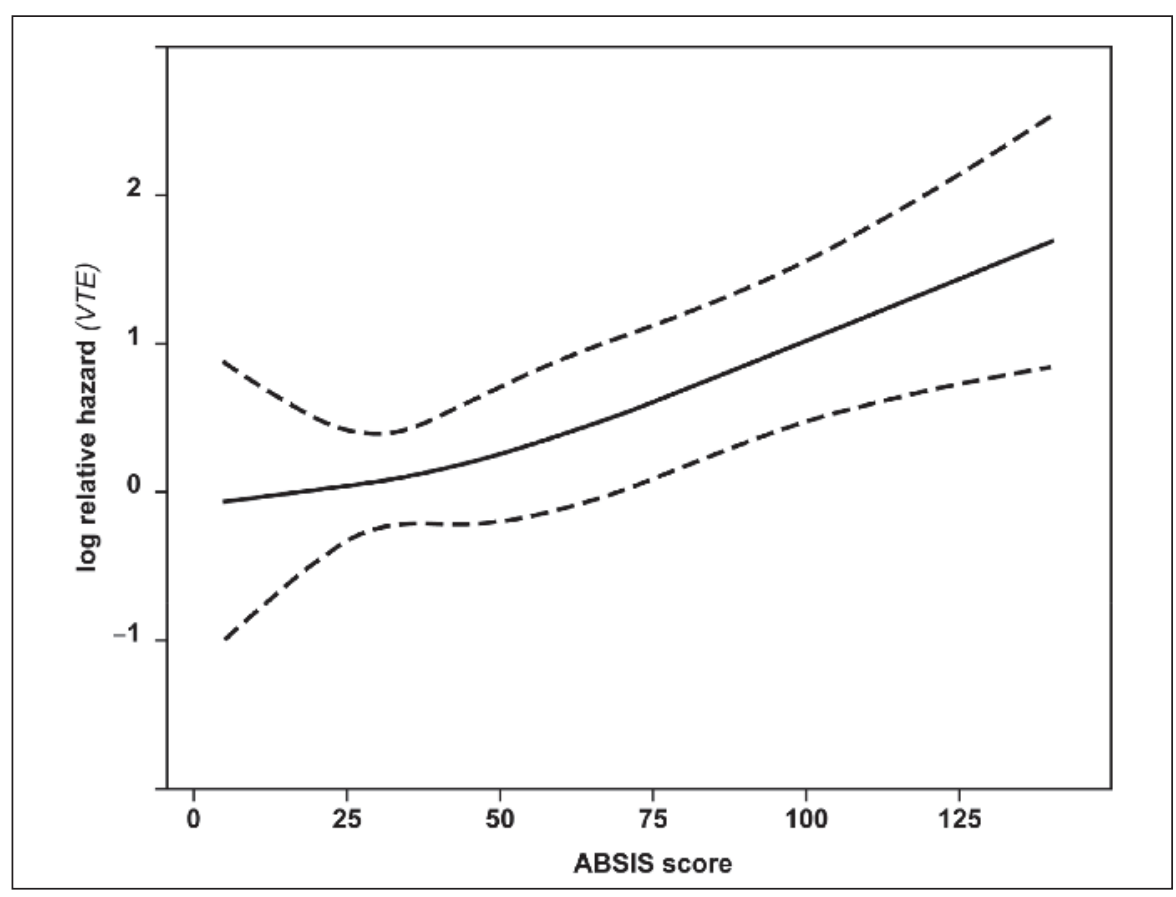

Figure 3: Restricted cubic spline curve showing the model-predicted log relative hazard of venous thromboembolism (VTE) against autoimmune bullous skin disorder intensity score (ABSIS) levels. Results are adjusted for age and sex in a Cox proportional hazard model. The dashed lines represent $95 \%$ confidence intervals. The non-linear component of the curve was not significant (Wald test: $p=0.605$ ). 
population according to age and sex ( Table 2), the standardised incidence ratio was 4.06 (95\%CI: 2.73-5.65), and was much higher in the acute phase (14.86 (95\%CI: 9.20-21.88)) than during remission (1.48 (95\%CI: 0.66-2.63)).

A linear relationship between ABSIS and the log of the relative hazard of VTE was found ( $>$ Figure 3; Wald test, $p=0.605$ for the non-linear component). The linear rise on a logarithmic scale corresponds to an exponential rise on a non-logarithmic scale. For every 10-point increase of ABSIS, the risk of VTE increased by $15 \%$ (age- and sex-adjusted HR, 1.15 (95\% CI: 1.06-1.25)). Compared to BP patients with ABSIS $<28$, those with ABSIS $>48$ had an almost three-fold increased risk of VTE (adjusted HR, 2.74 (95\% CI: 1.07-7.04), Table 3). The cumulative incidence of VTE at four years from BP diagnosis was 5\% (95\%CI: 1-9\%) for patients with ABSIS <28, 9\% (95\%CI: 3-14\%) for patients with ABSIS between 28 and 48 and 12\% (95\% CI: 7-17\%) for those with ABSIS $>48$.

About two thirds of the BP patients had not been exposed to any concomitant risk factor ( Table 1 ). Forty-four patients had been exposed to two or more risk factors. No patient had been exposed more than once to a transient risk factor. Of the 31 VTE episodes, 16 (52\%, of whom 13 during acute phase) occurred without concomitant risk factors, nine (29\%, 6 in acute phase) were related to one risk factor (transient in 5 and permanent in 4), and six (19\%, 3 in acute phase) occurred in the presence of $\geq 2$ risk factors (all transient in 3 cases, 1 transient and 1 permanent in 2 cases, and both permanent in 1 case). The risk of VTE was 2.5 times higher in patients sharing two or more risk factors than in those with no risk factor (adjusted HR, 2.56 (95\%CI: 1.00-6.70), $>$ Table $3)$. There was a multiplicative interaction between ABSIS and concomitant risk factors, as indicated in Table 4: patients with ABSIS $>48$ and $\geq 2$ risk factors had a six-fold higher risk of VTE
Table 3: Hazard ratio of VTE according to autoimmune bullous skin disorder intensity score (ABSIS) and presence of concomitant risk factors.

\begin{tabular}{l|l|l|l|}
\hline \multirow{2}{*}{ Variables } & \multicolumn{2}{l}{ VTE } & \multirow{2}{*}{ Hazard ratio $(95 \% \mathrm{CI})^{\dagger}$} \\
\cline { 2 - 3 } & no & yes & \\
\hline ABSIS * & & & 1 (Reference) \\
\hline$<28$ & 136 & 6 & $1.81(0.64-5.15)$ \\
\hline $28-48$ & 106 & 9 & $2.74(1.07-7.04)$ \\
\hline$>48$ & 143 & 16 & \\
\hline Concomitant risk factors & & 1 (Reference) \\
\hline 0 & 263 & 16 & $1.45(0.64-3.31)$ \\
\hline 1 & 100 & 9 & $2.56(1.00-6.70)$ \\
\hline$\geq 2$ & 38 & 6 & \\
\hline
\end{tabular}

${ }^{*}$ Data not available for 16 patients. ${ }^{\dagger}$ adjusted one for each other and for age and sex in a multivariable Cox proportional hazard model. $\mathrm{Cl}=$ confidence interval.

Table 4: Interaction between autoimmune bullous skin disorder intensity score (ABSIS) and concomitant risk factors on the risk of VTE.

\begin{tabular}{l|l|l|l}
\hline Groups at risk & VTE & $\begin{array}{l}\text { Hazard ratio } \\
(95 \% \mathrm{CI})^{\dagger}\end{array}$ \\
\cline { 2 - 4 } & no & yes & 1 (Reference) \\
\hline $\mathrm{ABSIS} \leq 48 \& \mathrm{RF}=0$ or 1 & 211 & 12 & $1.77(0.81-3.89)$ \\
\hline $\mathrm{ABSIS}>48 \& \mathrm{RF}=0$ or 1 & 136 & 13 & $1.82(0.51-6.47)$ \\
\hline $\mathrm{ABSIS} \leq 48 \& \mathrm{RF} \geq 2$ & 31 & 3 & $6.17(1.73-22.01)$ \\
\hline $\mathrm{ABSIS}>48 \& \mathrm{RF} \geq 2$ & 7 & 3 &
\end{tabular}

${ }^{*}$ ABSIS not available for 16 patients. ${ }^{\dagger}$ adjusted for age and sex in a multivariable Cox proportional hazard model. $\mathrm{RF}=$ risk factor; $\mathrm{Cl}=$ confidence interval.
Figure 4: Graphical representation of the interaction between autoimmune bullous skin disorder intensity score (ABSIS) and concomitant risk factors on the risk of venous thromboembolism (VTE). ABSIS is kept linear and concomitant risk factors are dichotomised [0 or 1 (dashed line), $\geq 2$ (solid line)]. VTE risk, adjusted for age and sex, is expressed as log of the relative hazard in a Cox proportional hazard model. The risk of VTE associated with ABSIS increases more sharply in individuals with $\geq 2$ concomitant risk factors, and the increased risk of VTE associated with the presence of $\geq 2$ risk factors is more evident for high levels of ABSIS. The absence of parallelism between the two lines indicates interaction ( $\beta$ estimate: 0.11 [95\% Cl: -0.04 to 0.27 ] for the interaction term).

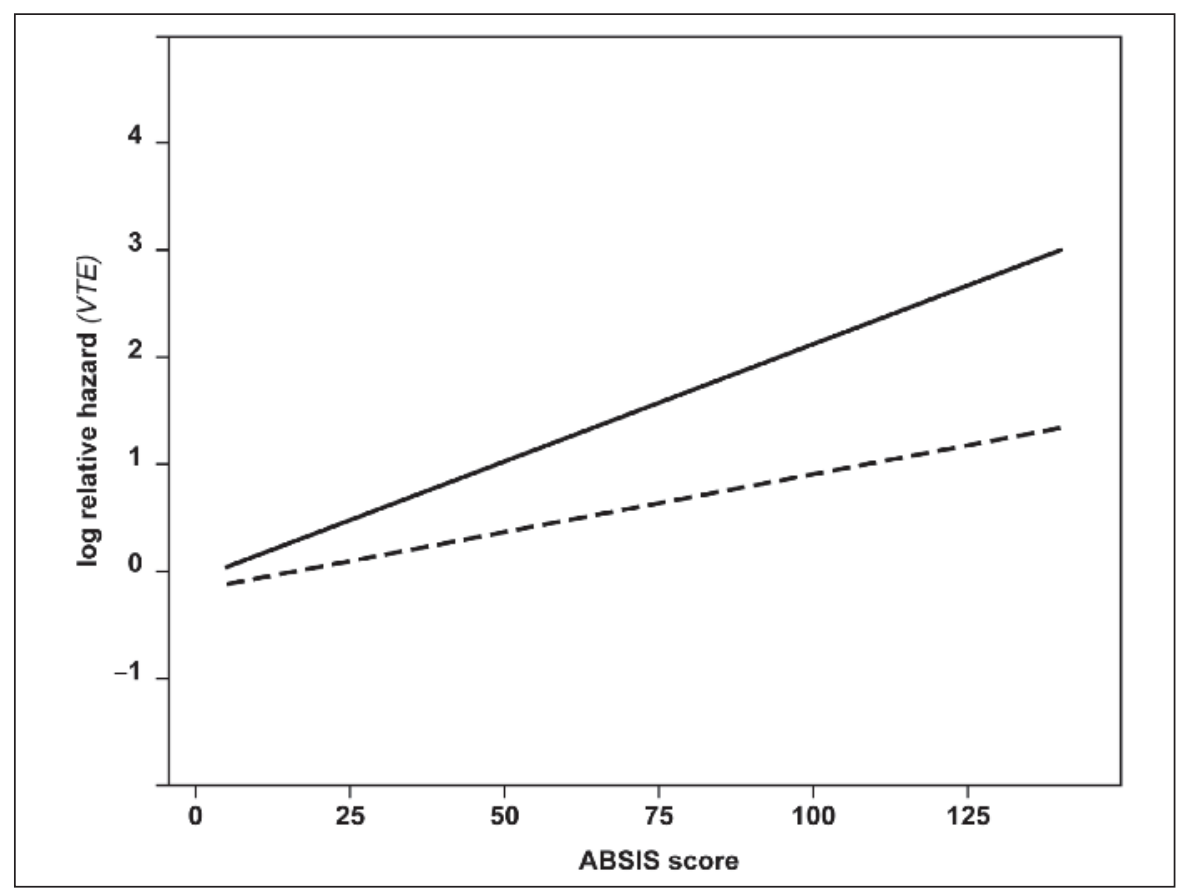


than those with ABSIS $\leq 48$ and one or no risk factor (adjusted HR: 6.17 (95\% CI: 1.73-22.01)). The interaction between ABSIS and concomitant risk factors can also be visually perceived in $>$ Figure 4 , which shows the linear increase of the log relative hazard of VTE according to ABSIS separately for BP patients with $\geq 2$ risk factors and for those with one or no risk factor ( $\beta$ estimate: 0.11 (95\% CI: -0.04 to 0.27 ) for the interaction term).

In a sensitivity analysis, where the 16 missing values for ABSIS were substituted with values obtained from linear interpolation by multiple imputation, none of the results on VTE risk changed.

\section{Discussion}

This multicentre cohort study shows that the risk of developing VTE is increased four-fold in patients with BP as compared with the general population of same age and sex. More specifically, the VTE risk increases up to 15 times during the acute phase of the disease (indicating a close link between the inflammatory state occurring in active BP and thrombosis), and drops to 1.5 times during clinical remission (indicating that a good control of the disease dramatically reduces the occurrence of thrombotic events). The tight connection between the inflammatory burden in BP patients and the risk of VTE is corroborated by the clear association between ABSIS and thrombotic risk, as the multivariable Cox regression analysis showed that for every 10-point increase of the ABSIS the risk of VTE increased by about $15 \%$. Several lines of evidence support an increased thrombotic risk in acute and chronic inflammatory disorders, such as sepsis (18), rheumatoid arthritis (19, 20 ), autoimmune skin diseases $(6,7)$, inflammatory bowel diseases (21), and hypereosinophilic syndromes (22). This may be explained, at least in part, by the increased expression of tissue factor, the main initiator of blood coagulation, induced by proinflammatory cytokines such as interleukin 6 (IL-6) and tumour necrosis

\section{What is known about this topic?}

- Bullous pemphigoid is a rare autoimmune blistering disease due to autoantibodies against two hemidesmosomal antigens. It mainly affects elderly and is progressively becoming an emerging condition with a high risk of death, due to sepsis and cardiovascular events.

- Bullous pemphigoid is characterised by marked activation of coagulation and to date there are no studies on the incidence of venous thromboembolism in this disease.

\section{What does this paper add?}

- The present multicentre cohort study on a large series of patients with bullous pemphigoid demonstrated a four-fold increased risk of venous thromboembolism which reached an increase of 15 folds during the acute phase of the disease.

- The high thrombotic tendency found in patients with bullous pemphigoid suggests a need for venous thromboembolism prophylaxis, particularly during the acute phase of the disease. factor alpha (TNF- $\alpha)$ (23). The strong expression of tissue factor by eosinophils, demonstrated in BP lesional skin (6), and the elevated levels of prothrombin fragment $\mathrm{F} 1+2$ and $\mathrm{D}$-dimer in both blister fluid and plasma $(6,7,10)$ indicate an activation of coagulation in BP patients. Coagulation activation generates thrombin, which may play a role at local level, by increasing vascular permeability $(24,25)$, and at systemic level, by increasing thrombotic risk.

Of particular interest was the interaction between ABSIS and concomitant risk factors on the subsequent risk of VTE. Patients with a high ABSIS and carrying two or more risk factors had a much higher risk of VTE than those with only one of the two conditions, indicating a possible synergistic effect. Considering the potential impact of genetic risk factors and hormonal treatment on the risk of VTE, we can say that no patient was taking hormonal compounds and that the old age of the patients and the absence of previous VTE episodes made the search for inherited thrombophilic defects not mandatory.

The results of our study imply some practical considerations. The high thrombotic tendency found in patients with BP, particularly evident during the acute phase of the disease, suggests a need for studies on the efficacy and safety of VTE prophylaxis in BP with antithrombotic drugs in this phase. Indeed, the $6 \%$ absolute risk of VTE found in the acute phase (incidence rate, 56.7 per 1,000 patient-years) outweights the absolute risk of major bleeding during extended-duration thromboprophylaxis in acutely-ill medical patients (less than 1\%) (26).

Strengths of this study are its large sample size relative to the rarity of the disorder, the low frequency of loss to follow-up and the wide panel of clinical parameters evaluated. Due to the rarity of the exposure (BP), its relationship with the VTE risk can only be addressed with a cohort study, as other approaches (i.e. casecontrol or case-cohort studies) would not be feasible.

The main limitation of this study is that we could not establish the exact cause of death in some of the 92 patients who died during the follow-up. In 36 cases the cause was not specified, and in 15 the reported cause was heart failure. The possibility that some of these deaths were related to pulmonary embolism can not be ruled out. However, this would have led to an underestimation of the true risk of VTE associated with BP, thus reinforcing our finding of a strong association between BP and risk of VTE. The possibility that only severe cases of BP would have been included in this study can be reasonably ruled out because the diagnosis of BP is based on laboratory investigations performed only in referral centres, and all patients (whatever the severity of the disease) are followed by the same centres after diagnosis. Another possible limitation is that, in the absence of data on the VTE incidence in the general Italian population, we standardised VTE rates of our Italian cohort of BP patients by using rates of a large Norwegian population as standard (17). However, this is not a major source of bias, because the incidence rates of VTE in Caucasians in the industrialised world do not differ much over country and over time $(17,27)$. To further verify our findings, we have redone calculations of standardised incidence rates during the acute phase of BP using VTE rates of a French population as standard (27), and found a standardised incidence ratio of 11.6 (95\%CI: 7.0-16.6), similar to 
that found by using Norwegian data. Steroid use was not included in the multivariable Cox model because all BP patients were given the same scheduled steroid treatment after BP diagnosis. Previous publications (28) revealed an increased risk for VTE under steroids. Our data show that this correlation is probably due to active disease phases in which steroids are administered rather than to pharmacological properties of steroids.

In conclusion, this study showed that patients with BP have a four-fold increased risk of VTE compared to individuals of the general population of same age and sex. This risk is much higher during the acute phase (up to 15 -folds) and is directly proportional to the disease intensity. The presence of concomitant risk factors further increases the risk of VTE.

\section{Author contributions}

$\mathrm{MC}$ and AVM designed the study and contributed equally to this article. MC, AVM, YB, GC, PQ, PCP, MC, MA, CDS, AP, EC, MP, AT, CC and the investigators of the INVENTEP Study Group followed the patients and collected clinical and laboratory data. MC, AVM, PB and FRR organized and evaluated the data. PB and FRR performed the statistical analysis and all the authors contributed in the interpretation of the results. MC and AVM drafted the manuscript; PB and FRR contributed in writing. All the authors critically reviewed the manuscript and approved the final version for submission.

\section{Conflicts of interest}

None declared.

\section{References}

1. Yancey KB. The pathophysiology of autoimmune blistering diseases. J Clin Invest 2005; 115: 825-828.

2. Langan SM, Smeeth L, Hubbard R, et al. Bullous pemphigoid and pemphigus vulgaris-incidence and mortality in the UK: population based cohort study. Br Med J 2008; 337: a180.

3. Roujeau JC, Lok C, Bastuji-Garin S, et al. High risk of death in elderly patients with extensive bullous pemphigoid. Arch Dermatol 1998; 134: 465-469.

4. Marazza G, Pham HC, Schärer L, et al. Autoimmune bullous disease Swiss study group. Incidence of bullous pemphigoid and pemphigus in Switzerland: a 2-year prospective study. Br J Dermatol 2009; 161: 861-868.

5. Hertl M, Eming R, Veldman C. T cell control in autoimmune bullous skin disorders. J Clin Invest 2006; 116: 1159-1166.

6. Marzano AV, Tedeschi A, Fanoni D, et al. Activation of blood coagulation in bullous pemphigoid: role of eosinophils, and local and systemic implications. Br J Dermatol 2009; 160: 266-272.

7. Marzano AV, Tedeschi A, Spinelli D, et al. Coagulation activation in autoimmune bullous diseases. Clin Exp Immunol 2009; 158: 31-36.
8. Cugno M, Tedeschi A, Asero R, et al. Skin autoimmunity and blood coagulation. Autoimmunity 2010; 43: 189-194.

9. Cugno M, Tedeschi A, Crosti C, et al. Activation of blood coagulation in autoimmune skin disorders. Exp Rev Clin Immunol 2009; 5: 605-613.

10. Marzano AV, Tedeschi A, Berti E, et al. Activation of coagulation in bullous pemphigoid and other eosinophil-related inflammatory skin diseases. Clin Exp Immunol 2011; 165: 44-50.

11. Marzano AV, Tedeschi A, Polloni I, et al. Prothrombotic state and impaired fibrinolysis in bullous pemphigoid, the most frequent autoimmune blistering disease. Clin Exp Immunol 2013; 171: 76-81.

12. Rosendaal FR, Van Hylckama Vlieg A, Doggen CJ. Venous thrombosis in the elderly. J Thromb Haemost 2007; 5 (Suppl 1):310-317.

13. Joly P, Benichou J, Lok C, et al. Prediction of survival for patients with bullous pemphigoid: a prospective study. Arch Dermatol 2005; 141: 691-698.

14. Yang YW, Chen YH, Xirasagar S, et al. Increased risk of stroke in patients with bullous pemphigoid: a population-based follow-up study. Stroke 2011; 42: 319-323.

15. Langan SM, Hubbard R, Fleming K, et al. A population-based study of acute medical conditions associated with bullous pemphigoid. Br J Dermatol 2009; 161: 1149-1152.

16. Pfutze M, Niedermeier A, Hertl M, et al. Introducing a novel autoimmune bullous skin disorder intensity score (ABSIS) in pemphigus.Eur J Dermatol 2007; 17: 4-11.

17. Naess IA, Christiansen SC, Romundstad P, et al. Incidence and mortality of venous thrombosis: a population-based study. J Thromb Haemost 2007; 5: 692-699.

18. Levi M. The coagulant response in sepsis and inflammation. Hamostaseologie 2010; 30: 10-16.

19. Ingegnoli F, Fantini F, Favalli EG, et al. Inflammatory and prothrombotic biomarkers in patients with rheumatoid arthritis: effects of tumor necrosis factoralpha blockade. J Autoimmun 2008; 31: 175-179.

20. Ingegnoli F, Fantini F, Griffini S, et al. Anti-tumor necrosis factor alpha therapy normalizes fibrinolysis impairment in patients with active rheumatoid arthritis. Clin Exp Rheumatol 2010; 28: 254-257.

21. Irving PM, Pasi KJ, Rampton DS. Thrombosis and inflammatory bowel disease. Clin Gastroenterol Hepatol 2005; 3: 617-628.

22. Ogbogu PU, Rosing DR, Horne MK 3rd. Cardiovascular manifestations of hypereosinophilic syndromes. Immunol Allergy Clin North Am 2007; 27: 457-475.

23. Levi M, van der Poll T. Two-way interactions between inflammation and coagulation. Trends Cardiovasc Med 2005; 15: 254-259.

24. Garcia JG, Siflinger-Birnboim A, Bizios R, et al. Thrombin-induced increase in albumin permeability across the endothelium. J Cell Physiol 1986; 128: 96-104.

25. DeMichele MA, Moon DG, Fenton JW 2nd, et al. Thrombin's enzymatic activity increases permeability of endothelial cell monolayers. J Appl Physiol 1990; 69: 1599-1606.

26. Hull RD, Schellong SM, Tapson VF, et al., for the EXCLAIM study. Extendedduration venous thromboembolism prophylaxis in acutely ill medical patients with recently reduced mobility. Ann Intern Med 2010; 153: 8-18.

27. Oger E, for the EPI-GETBO Study Group. Incidence of venous thromboembolism: a community-based study in Western France. Thromb Haemost 2000; 83: 657-660.

28. Johannesdottir SA, Horváth-Puhó E, Dekkers OM, et al. Use of glucocorticoids and risk of venous thromboembolism. A nationwide population-based casecontrol study. J Am Med Assoc Intern Med 2013; 173: 743-752. 